\title{
Familiäre pulmonale Zysten und papulöse Haut- effloreszenzen bei einer 37-jährigen Patientin
}

\author{
Familial Pulmonary Cysts and Papular Skin Lesions in a 37-Year-Old Woman
}

Autoren

Institute
K. E. Sander ${ }^{1}$, P. A. Schnabel ${ }^{2,3,4}$, C. P. Heußel ${ }^{3,4,5}$, J. Pfannschmidt ${ }^{6}$, F. J. Herth'1,3,4 M. Kreuter $^{1,3,4}$

Die Institutsangaben sind am Ende des Beitrags gelistet. eingereicht 4.6 .2013

akzeptiert 10.6.2013

\section{Bibliografie}

DOI http://dx.doi.org/

10.1055/s-0033-1344350

Online-Publikation: 11.7.2013

Pneumologie 2013; 67: 471-475

(c) Georg Thieme Verlag KG

Stuttgart · New York

ISSN 0934-8387

\section{Korrespondenzadresse}

PD Dr. Michael Kreuter

Pneumologie und

Beatmungsmedizin, Thoraxklinik

Universitätsklinikum Heidelberg

Amalienstr. 5

69126 Heidelberg

michael.kreuter@thoraxklinik-

heidelberg.de

\section{Zusammenfassung \\ $\nabla$}

Eine 37-jährige Patientin stellte sich mit plötzlich aufgetretener Luftnot und thorakalen Schmerzen vor. Bei der Patientin und bei zwei Familienmitgliedern hatten sich in der Vergangenheit bereits mehrmals Spontanpneumothoraces ereignet. Die Patientin wies zudem papulöse Hauteffloreszenzen im Gesicht auf. Radiomorphologisch sah man einen Pneumothorax links apikal und basal betonte zystische Lungenveränderungen beidseits. Therapeutisch erfolgten eine Pleurodese und mehrere Keilresektionen mit Einlage einer Drainage links. Histologisch fanden sich multiple Zysten, wobei immunhistochemisch typische Differenzialdiagnosen ausgeschlossen werden konnten. Eine molekulargenetische Untersuchung wies eine heterozygote Mutation des Follikulin (FLCN)-Gens nach. Somit wurde ein Birt-HoggDubé Syndrom (BHDS) diagnostiziert. Das BHDS unterliegt einem autosomal dominanten Erbgang und zeichnet sich durch zystische Lungenveränderungen mit rezidivierenden Pneumothoraces, kutanen Fibrofollikulomen und einem erhöhten Risiko für Nierenzellkarzinome aus. Es geht mit Mutationen im für das Protein FLCN kodierenden Gen auf Chromosom 17 einher.

\section{Einleitung}

\section{$\nabla$}

Eine pulmonale Zyste ist eine mit Luft gefüllte Struktur, die eine dünne Wand umgibt. Zystischen Lungenveränderungen können unterschiedliche Erkrankungen zugrunde liegen. Dazu gehören Kollagenosen und Vaskulitiden, die mit einer fibrosierenden Alveolitis einhergehen, und seltene interstitielle Lungenerkrankungen wie die pulmonale Langerhans-Zell-Histiozytose (PLCH), die Lymphangioleiomyomatose (LAM) und die lymphozytäre interstitielle Pneumonie (LIP). Darüber hinaus können Infektionen mit bestimmten Bakterien und Pilzen, wie beispielswei-

\section{Abstract \\ $\nabla$}

A 37-year-old female patient presented with sudden dyspnea and chest pain. Spontaneous pneumothoraces had been observed several times before in this patient and two members of the patient's family in the last years. Moreover, she exhibited papular facial skin lesions. Radiomorphologically a pneumothorax apical on the left side and basal accentuated cystic lung destruction on both sides could be seen. Pleurodesis and several wedge resections with insertion of a drainage on the left side were performed therapeutically. Histology disclosed multiple cysts, whereby typical differential diagnoses could be excluded by immunohistochemistry. A molecular genetic investigation detected a heterozygous mutation in the gene coding for follikulin (FLCN). Thereby, Birt-Hogg-Dubé syndrome (BHDS) was diagnosed. BHDS follows autosomal dominant inheritance and is characterized by cystic lung lesions with recurrent pneumothoraces, cutaneous fibrofolliculomas and an increased risk of renal carcinomas. It is based on mutations in the gene coding for the protein FLCN on chromosome 17.

se Pneumocystis jirovecii, Metastasen, eine Sarkoidose, eine Amyloidose und selten eine desquamative interstitielle Pneumonie (DIP) zu zystischen Veränderungen des Lungenparenchyms führen. Eine weitere Ursache pulmonaler Zysten ist das Birt-Hogg-Dubé Syndrom (BHDS), eine Genodermatose, die sich gewöhnlich im Bereich der Haut, der Nieren und der Lunge manifestiert. Weltweit sind etwa 200 betroffene Familien bekannt [1]. Das BHDS, auch Hornstein-Birt-HoggDubé Syndrom genannt, zeichnet sich durch die klinische Trias zystische Lungendegeneration mit konsekutivem Pneumothorax, kutane Fibrofollikulome und renale Karzinome aus [2,3,4]. 


\section{Kasuistik}

$\nabla$

Anamnese

Eine 37-jährige Patientin stellte sich mit Luftnot, Husten und seit einigen Stunden bestehenden Schmerzen linksthorakal und linksdorsal ambulant vor. In den letzten vier Jahren hatten sich bereits mehrere Spontanpneumothoraces rechts ereignet, wobei unter anderem eine Video-assistierte Thorakoskopie (VATS) rechts mit Talkumpleurodese durchgeführt worden war. Kurz darauf zeigte sich erneut zweimal eine Lungenablösung der gleichen Seite, welche jeweils konservativ ohne Einlage einer Drainage therapiert wurde. In der Familienanamnese berichtete die Patientin, dass sowohl bei ihrem Vater als auch bei einem Onkel väterlicherseits in der Vergangenheit wiederholt Pneumothoraces aufgetreten seien. Die Patientin ist Kaukasierin und hat keine Exposition gegenüber Gefahrenstoffen am Arbeitsplatz oder in der Freizeit. Die Reiseanamnese war ebenfalls unauffällig. Es bestanden keine Vorerkrankungen, außer einer Pollenallergie. Die Patientin gab weder Dauermedikation noch Nikotinabusus an.

\section{Diagnostik und Therapie}

Bei der körperlichen Untersuchung zeigten sich reizlose Wundverhältnisse rechts thorakal bei Zustand nach VATS sowie mehrere papulöse Hauteffloreszenzen im Gesicht $(\bullet$ Abb. 1). Diese traten vor etwa drei Jahren erstmalig auf und sind seitdem unverändert. Bei der Auskultation der Lunge fiel ein hypersonorer Klopfschall links apikal auf. Die laborchemischen Werte waren normal. Elektrokardiographisch wurde ein unauffälliger Befund erhoben. In der Blutgasanalyse sah man Zeichen einer Hyperventilation $\left(\mathrm{pO}_{2} 86 \mathrm{mmHg}, \mathrm{pCO}_{2} 33 \mathrm{mmHg}\right.$ ) bei noch ausgeglichenem $\mathrm{pH}-$ Wert. Von einer weiteren Lungenfunktionsdiagnostik wurde bei Verdacht auf Pneumothorax Abstand genommen. Die RöntgenThorax-Aufnahme in zwei Ebenen zeigte eine Ablösung links apikal ( Abb.2). Es wurde auch unter Berücksichtigung der Vorgeschichte daraufhin eine Computertomografie des Thorax durchgeführt, welche einen $1 \mathrm{~cm}$ messenden Mantelpneumothorax links sowie einen diskreten gekammerten Pneumothorax im rechten Randwinkel nachwies. Zudem sah man vor allem in den basalen Lungenabschnitten subpleural gelegene, sehr dünnwandige zystische Lungenveränderungen ( $\mathbf{A b b}$.3a, b).

Aufgrund der Vorgeschichte wurde bei nun erstmaligem Ereignis eines sekundären Spontanpneumothorax links die Indikation zur VATS links mit Keilresektionen in Segment 5 und 8, Talkumpleurodese und Drainageneinlage gestellt. Intraoperativ wurden mehrere Bullae abgetragen. Einzelne subpleurale Blasen und weitere tief im Parenchym liegende bullöse Veränderungen wurden belassen, da bei der insgesamt stark destruierten Lunge nach Resektion eine hohe Fistelneigung zu erwarten war. In der histologischen Untersuchung des entnommenen Gewebes wurden eine pleurale und subpleural akzentuierte Fibrose sowie multiple Zysten gesehen ( Abb.4a,b). Typische Zellformationen bzw. Veränderungen, die bei einer differenzialdiagnostisch in Erwägung gezogenen LAM oder einer PLCH nachzuweisen sind, lagen nicht vor. Die vorliegenden Befunde wurden daraufhin in unserem interdisziplinären Board für seltene und interstitielle Lungenerkrankungen diskutiert.

Aufgrund der Hautläsionen wie auch der familiären Häufung der Pneumothoraces wurde differenzialdiagnostisch ein BHDS in Betracht gezogen und eine molekulargenetische Mutationsanalyse des Follikulin (FLCN)-Gens vorgenommen. Die Untersuchung deckte eine heterozygote Mutation auf.

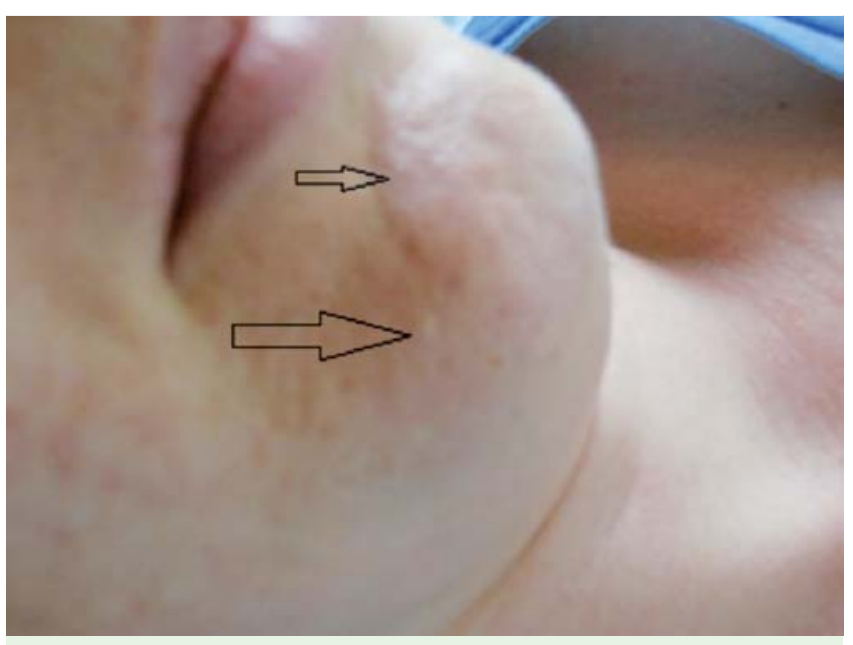

Abb. 1 Mehrere hautfarbene papulöse Hautläsionen (Fibrofollikulomen entsprechend) der Patientin im Bereich des Kinns (Pfeile=Fibrofollikulome).

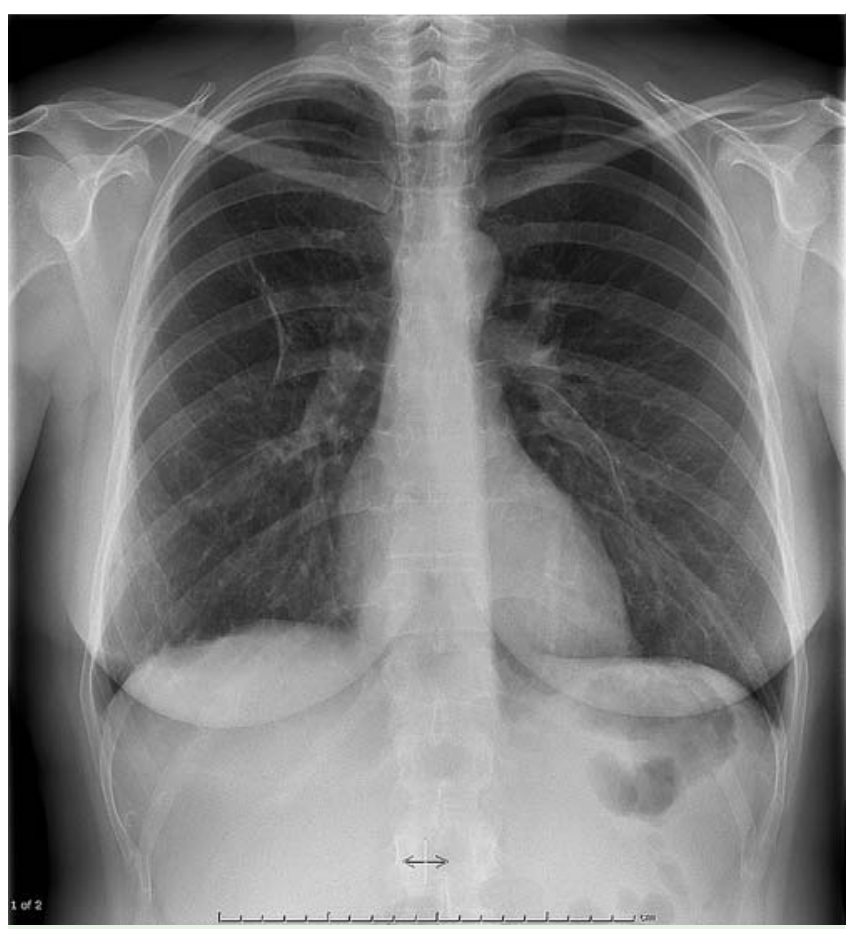

Abb.2 Röntgenaufnahme des Thorax mit Nachweis eines Pneumothorax links apikal. Man erkennt zudem eine schmale, glatt begrenzte längliche Verschattung in beiden Mittelfeldern, möglicherweise Plattenatelektasen entsprechend.

Ferner erfolgte eine Magnetresonanztomografie der Nieren zum Ausschluss einer Neoplasie mit unauffälligem Befund ( $\bullet$ Abb.5). Der postoperative Verlauf gestaltete sich komplikationslos. Der weitere klinische Verlauf ist seither unauffällig.

\section{Diskussion}

$\nabla$

Mit Luft gefüllte Hohlräume innerhalb des Lungenparenchyms werden radiologisch als Zysten oder Kavernen klassifiziert. Erstgenannte schließen die Subgruppen Bullae, Blebs und Pneumatozelen ein. Sie unterscheiden sich in ihrer Form, dem Wanddurch- 

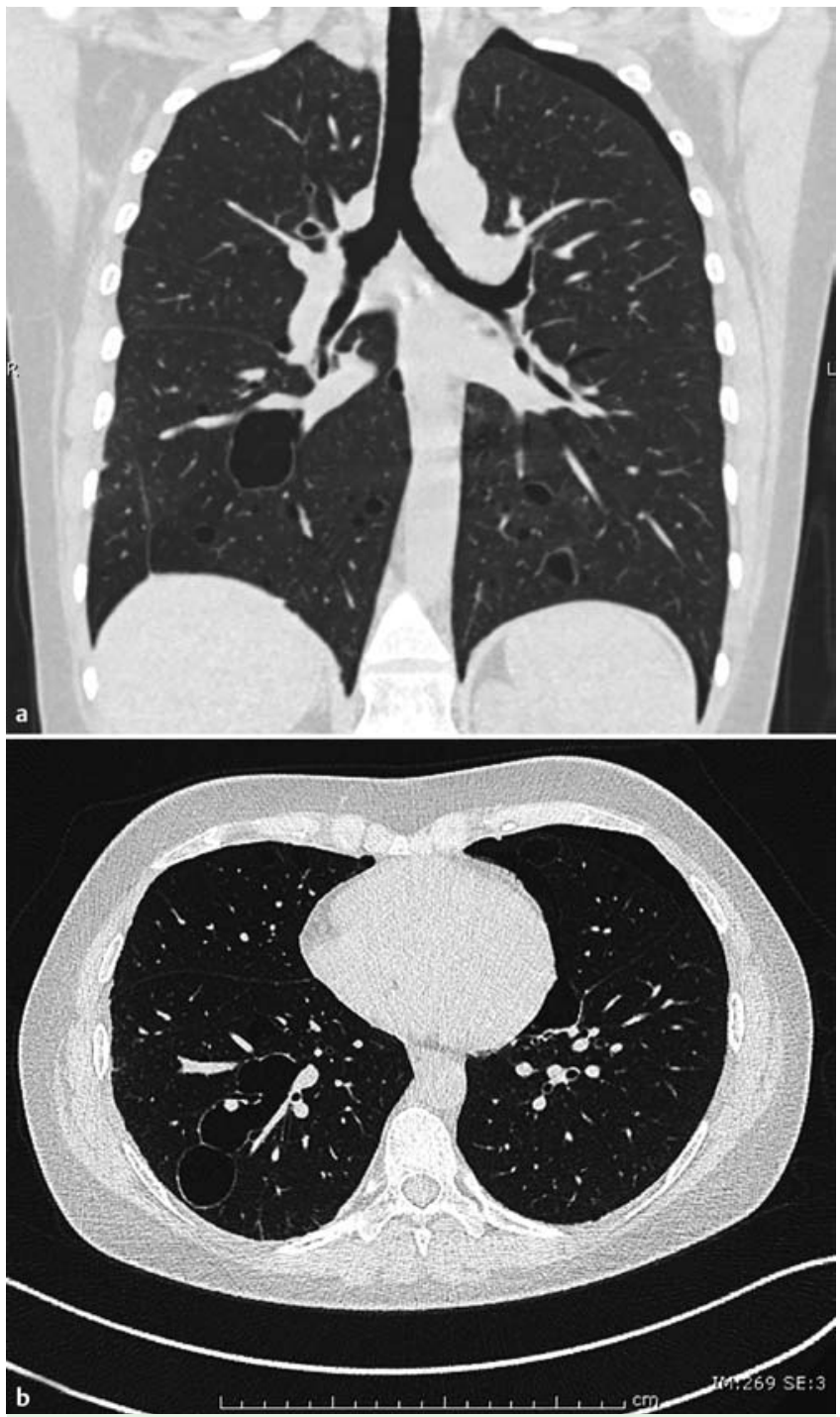

Abb.3 a, b Die Computertomografie des Thorax mit koronarer Reformatierung zeigt einen $1 \mathrm{~cm}$ messenden Mantelpneumothorax links und vor allem basal gelegene multiple dünnwandige Lungenzysten.

messer, der Größe, der Anzahl und dem Verteilungsmuster im Lungenparenchym. Zysten besitzen eine scharf abgegrenzte dünne Wand $(<4 \mathrm{~mm})$, wohingegen Kavernen von einer dickeren Wand ( $>4 \mathrm{~mm}$ ) umgeben sind. Beide Entitäten müssen von radiomorphologisch ähnlichen Strukturen wie dem Emphysem, der Lungenfibrose mit Honeycombing, lokal begrenzten Pneumothoraces und der Bronchiektasie unterschieden werden, um eine korrekte Diagnosestellung und Therapie zu gewährleisten [5].

Die PLHC und die LAM sind wichtige Differenzialdiagnosen des BHDS und zählen zu den diffusen zystischen Lungenerkrankungen. Die PLHC zeichnet sich durch die Akkumulation pathologischer Langerhans-Zellen in einem oder mehreren Organen aus, wobei ca. $40 \%$ der Betroffenen eine Lungenbeteiligung aufweisen [6]. Die Erkrankung ist selten und findet sich hauptsächlich bei jungen Erwachsenen mit Nikotinabusus. Radiomorphologisch sieht man häufig bizarr geformte Zysten vorwiegend in den Oberlappen. Eine Aussparung der kostophrenischen Winkel ist typisch. Die Zysten sind dünnwandig und gehen häufig mit peribronchialen Noduli einher.

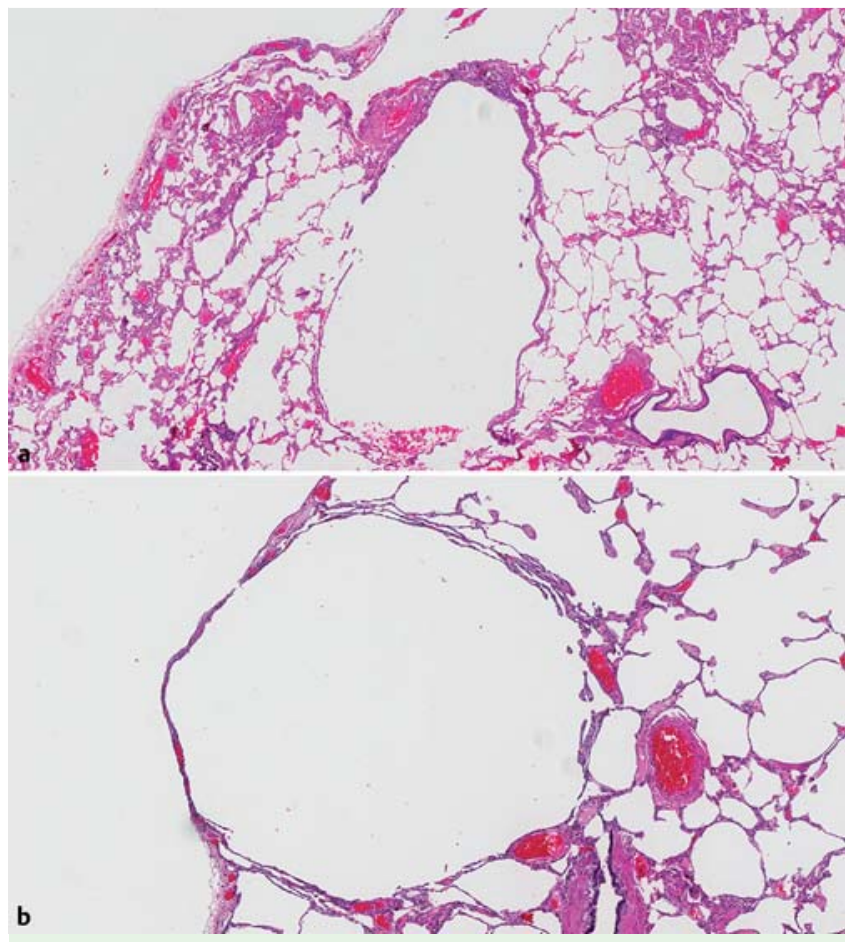

Abb. 4 a, b Lungenkeilresektate aus S5 und S8 links zeigen eine pleurale und subpleural akzentuierte, teils auch peribronchiale und perivaskuläre Fibrose sowie multiple, $0,3 \mathrm{~cm}$ bis $2,2 \mathrm{~cm}$ durchmessende Zysten, die von einer teilweise leicht verdickten Wand mit einer dünnen epithelialen Zellschicht ausgekleidet sind. Des Weiteren sieht man eine moderate Mediahypertrophie muskulärer und kleiner elastischer Pulmonalarterienäste mit perivaskulärer Fibrose. (a) Hämatoxylin-Eosin-Färbung (HE), Primärvergr. 1,8x (b) HE, Primärvergr. $3 \mathrm{x}$.

Die LAM ist charakterisiert durch die gesteigerte Proliferation atypischer glatter Muskelzellen in der Lunge, den Nieren und dem lymphatischen System. Sie betrifft hauptsächlich Frauen im gebärfähigen Alter und tritt sporadisch oder in Assoziation mit tuberöser Sklerose auf [5]. Bei Patienten mit LAM findet man eine pathognomonische Morphologie mit unterschiedlich großen, dünnwandigen Zysten, die über die gesamte Lunge verteilt sind, und häufig eine Erweiterung des Ductus thoracicus. Patienten mit tuberöser Sklerose weisen zudem häufig Angiofibrome im Gesicht und renale Tumore auf, weshalb eine Abgrenzung zu anderen Syndromen, wie dem BHDS, nicht immer einfach ist.

Pulmonale Zysten bei BHDS befinden sich vorwiegend in den basalen Lungenabschnitten. Konventionelle Röntgenaufnahmen des Thorax sind häufig unauffällig [6]. Die autosomal-dominant vererbbare Erkrankung entsteht durch unterschiedliche Mutationen im für FLCN kodierenden Gen [7]. Dieses befindet sich auf Chromosom 17. Das Protein FLCN ist in unterschiedlichen Geweben lokalisiert, darunter auch in der Haut und im Lungen- und Nierenparenchym [8]. FLCN bildet einen Komplex mit den Proteinen FNIP1 und FNIP2, der wiederum mit Signalmolekülen wie AMPK und mTOR interagiert. Es ist anzunehmen, dass FLCN als Tumorsuppressor wirkt und die renale Karzinogenese hemmt $[9,10]$.

1975 beschrieben Hornstein et al. erstmals multiple Hautläsionen und zur Entartung neigende Kolonpolypen bei zwei Geschwistern, deren Vater ähnliche Hauteffloreszenzen sowie Nieren- und Lungenzysten aufwies [11]. Zwei Jahre später veröffentlichten Birt et al. einen Artikel über das gehäufte Auftreten 


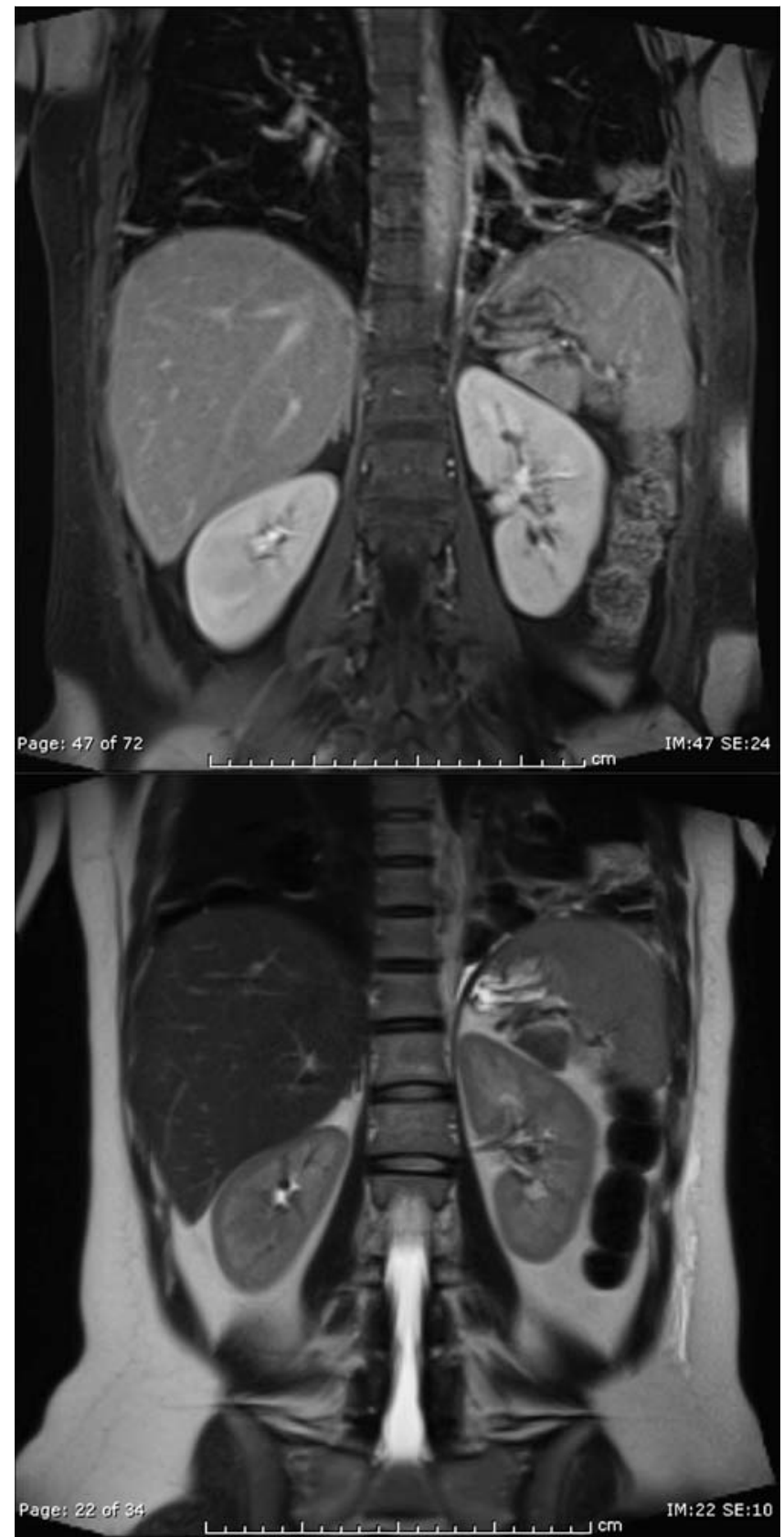

Abb.5 Magnetresonanztomografie des Oberbauchs ohne Hinweis auf einen Nierentumor oder malignomsuspekt vergrößerte retroperitoneale Lymphknoten. Postoperativ zeigen sich links eine Unterlappenteilatelektase und ein diskreter Pleuraerguss.

papulöser Hauttumore, so genannter Fibrofollikulome, in einer Familie [12].

\section{Haut}

Bei Patienten mit BHDS werden histologisch häufig Fibrofollikulome, benigne Tumore der Haarfollikel, nachgewiesen. Sie erscheinen gewöhnlich in der dritten und vierten Lebensdekade und nehmen an Größe und Anzahl im Verlauf zu [8,13]. In der Regel befinden sich die kutanen Läsionen im Bereich der Nase, der Wangen, seltener am Hals, Oberkörper und den Ohren [14]. Sie erscheinen als multiple, etwa $2-4 \mathrm{~mm}$ große, weißliche Papeln [8]. Die Diagnose basiert sowohl auf dem klinischen Erscheinungsbild als auch auf der histologischen Untersuchung [14].

\section{Lunge}

Über $80 \%$ der erwachsenen BHDS-Patienten haben multiple pulmonale Zysten, die sich überwiegend in den basalen Lungenabschnitten befinden. Das übrige Lungenparenchym stellt sich zumeist unauffällig dar, was die oft nur geringfügig oder gar nicht eingeschränkte Lungenfunktion bei BHDS-Patienten erklärt [14]. Die genaue Pathogenese der zystischen Lungendegeneration ist bislang ungeklärt. Jedoch konnte gezeigt werden, dass FLCN in hohem Maße in Lungenfibroblasten und Makrophagen exprimiert wird. Mutationen im FLCN-Gen könnten so zu Verlagerungen im Gleichgewicht von Zytokinen und Proteasen führen, welches für die Integrität der extrazellulären Matrix im Lungenparenchym essentiell ist [15]. Der Spontanpneumothorax tritt bei BHDS-Patienten meistens rezidivierend auf [14]. In einer Studie mit 198 Patienten aus 89 BHDS-Familien hatten 24\% in der Vergangenheit einen Pneumothorax erlitten. Es wurde nachgewiesen, dass eine signifikante Korrelation zwischen Anzahl wie auch Größe der pulmonalen Zysten und dem Risiko für einen Pneumothorax besteht [16].

\section{Niere}

Eine weitere Komplikation des BHDS ist das erhöhte Risiko, an einem renalen Karzinom zu erkranken [17]. Es existieren unterschiedliche histologische Subtypen von Nierentumoren, die mit dem BHDS einhergehen. Dazu gehören vor allem chromophobe renale Karzinome, aber auch chromophob-onkozytäre Hybridtumore und klarzellige Nierenzellkarzinome. Die renalen Neoplasien treten häufig multipel und bilateral auf $[14,4,17]$. In einer Studie mit 115 FLCN Mutationsträgern wurde ein auf die gesamte Lebenszeit bezogenes Risiko von ca. 16\%, an einem Nierenkarzinom zu erkranken, ermittelt [18].

\section{Diagnose \\ $\nabla$}

Zur Diagnosestellung eines BHDS ist es nicht obligat, dass der Patient alle genannten klinischen Zeichen aufweist [19]. Gewöhnlich wird er aufgrund wiederholter Episoden von Pneumothoraces klinisch auffällig [9]. Eine Assoziation des BHDS sowohl mit kolorektalen Neoplasien als auch mit Schilddrüsentumoren, Nebenschilddrüsenadenomen, Lipomen und Sarkomen wird diskutiert $[8,18,17]$. Bei klinischem Verdacht auf BHDS sollte eine molekulargenetische Untersuchung in die Wege geleitet werden [19]. Dies ist bei Patienten mit frühzeitig auftretenden renalen Karzinomen ( $<50$ Jahre), besonders bei multifokalem und/oder bilateralem Auftreten, bei chromophober oder onkozytärer Histologie, bei Patienten mit rezidivierenden Pneumothoraces und unklarer basaler zystischer Lungenerkrankung der Fall [4]. Vor allem eine familiäre Häufung oben genannter Krankheitsbilder sollte eine molekulargenetische Diagnostik nach sich ziehen. Eine Mutationsanalyse ist selbst in klinisch unzweifelhaften Fällen bedeutsam, da sie unter anderem zur Identifikation und Beratung von gefährdeten Familienmitgliedern beitragen kann [14].

\section{Therapie}

$\nabla$

Eine ursächliche Therapie für das BHDS steht derzeit nicht zur Verfügung [15]. Fibrofollikulome können in manchen Fällen mittels Laserablation, Kürettage oder Elektrodesikkation entfernt werden, jedoch sind die Therapieoptionen noch begrenzt [14]. 
Die Behandlung eines BHDS-Patienten mit Pneumothorax entspricht der eines Patienten mit primärem Spontanpneumothorax. Da Zigarettenkonsum ein wichtiger Risikofaktor für die Entstehung eines Pneumothorax wie auch eines Nierenzellkarzinoms ist, müssen BHDS-Patienten mit Nikotinabusus eindringlich über die möglichen Folgen aufgeklärt werden [8]. Aufgrund des erhöhten Risikos für renale Karzinome sind regelmäßige Kontroll-Bildgebungen für Träger einer FLCN-Mutation und für Familienmitglieder mit klinisch vorhandenem BHDS indiziert, auch wenn bei diesen keine entsprechende Mutation nachgewiesen wurde $[14,18]$. Bisher gibt es keine klaren Empfehlungen bezüglich des Alters bei Beginn oder der Frequenz von abdominalen Screeninguntersuchungen für BHDS-Patienten. Es ist sinnvoll, frühzeitige, etwa mit dem 20. Lebensjahr einsetzende, jährliche Untersuchungen mittels Magnetresonanztomografie der Nieren durchzuführen $[8,14]$.

\section{Ausblick}

Die Identifikation von Proteinen, die mit FLCN zusammenwirken, wie auch die Kenntnis der Interaktion der daraus entstehenden Komplexe mit AMPK geben Aufschluss über eine Verbindung zwischen FLCN und der AMPK-mTOR Achse. Die Entwicklung von Medikamenten, die auf molekularer Ebene diese Signalwege beeinflussen, ist ein möglicher Ansatz in der Therapie der Fibrofollikulome und Nierenzellkarzinome bei BHDS-Patienten [10].

\section{Interessenkonflikt}

$\nabla$

Die Autoren geben an, dass kein Interessenkonflikt besteht. Der Beitrag von C. P. Heußel wurde unterstützt vom Deutschen Zentrum für Lungenforschung (DZL) durch Mittel des Bundesministeriums für Bildung und Forschung im Rahmen der Projekte 82DZL00401, 82DZL00402 und 82DZL00404.

\section{Institute}

${ }^{1}$ Pneumologie und Beatmungsmedizin, Thoraxklinik Universitätsklinikum Heidelberg

${ }^{2}$ Pathologisches Institut, Sektion Thoraxpathologie, Universitätsklinikum Heidelberg

${ }^{3}$ Zentrum für interstitielle und seltene Lungenerkrankungen, Zentrum für seltene Erkrankungen, Universitätsklinikum Heidelberg

${ }^{4}$ Deutsches Zentrum für Lungenforschung (DZL), Heidelberg (TLRC-H)

${ }^{5}$ Diagnostische und Interventionelle Radiologie mit Nuklearmedizin,

Thoraxklinik am Universitätsklinikum Heidelberg

${ }^{6}$ Thoraxchirurgie, Thoraxklinik Universitätsklinikum Heidelberg

\section{Literatur}

1 Mohan Das L, Rang CE, Banka R. Birt-Hogg-Dube syndrome: a rare cause of cystic lung diseases. BMJ Case Rep 2013: DOI 10.1136/bcr-2013008826

2 Nishii T, Tanabe M, Tanaka $R$ et al. Unique mutation, accelerated mTOR signaling and angiogenesis in the pulmonary cysts of Birt-Hogg-Dubé syndrome. Pathol Int 2013; 63: 45-55

3 Happle R. Hornstein-Birt-Hogg-Dubé syndrome: a renaming and reconsideration. Am J Med Genet 2012; 158A: 1247-1251

4 Pavlovich CP, Walther MM, Eyler RA et al. Renal tumors in the BirtHogg-Dubé syndrome. Am J Surg Pathol 2002; 26: 1542 - 1552

5 Cosgrove GP, Frankel SK, Brown KK. Challenges in pulmonary fibrosis. 3 : Cystic lung disease. Thorax 2007; 62: 820-829

6 Koo HK, Yoo CG. Multiple cystic lung disease. Tuberc Respir Dis 2013; 74: $97-103$

7 Toro JR, Wei MH, Glenn GM et al. BHD mutations, clinical and molecular genetic investigations of Birt-Hogg-Dubé syndrome: a new series of 50 families and a review of published reports. J Med Genet 2008; 45: $321-331$

8 Felton SJ, Madan V. Facial and upper body papules in a patient with a family history of recurrent pneumothorax. JAMA 2012; 308: 2622 2623

9 Furuya M, Nakatani Y. Birt-Hogg-Dubé syndrome: clinicopathological features of the lung. J Clin Pathol 2013; 66: 178-186

10 Schmidt LS. Birt-Hogg-Dubé syndrome: from gene discovery to molecularly targeted therapies. Fam Cancer 2012: DOI 10.1007/s10689012-9574-y

11 Hornstein OP, Knickenberg M. Perifollicular fibromatosis cutis with polyps of the colon - a cutaneo-intestinal syndrome sui generis. Arch Dermatol 1975; 253: $161-175$

12 Birt AR, Hogg GR, Dubé WJ. Hereditary multiple fibrofolliculomas with trichodiscomas and acrochordons. Arch Dermatol 1977; 113: 16741677

13 Starink TM, Houweling AC, van Doorn MB et al. Familial multiple discoid fibromas: a look-alike of Birt-Hogg-Dubé syndrome not linked to the FLCN locus. J Am Acad Dermatol 2012; 66: 259.e1 - e9

14 Menko FH, van Steensel MAM, Giraud S et al. Birt-Hogg-Dubé syndrome: diagnosis and management. Lancet Oncol 2009; 10: 1199-1206

15 Pimenta SP, Baldi BG, Nascimento EC et al. Birt-Hogg-Dubé syndrome: metalloproteinase activity and response to doxycycline. Clinics (Sao Paulo) 2012; 67: 1501 - 1504

16 Toro JR, Pautler SE, Stewart $L$ et al. Lung cysts, spontaneous pneumothorax, and genetic associations in 89 families with Birt-Hogg-Dubé syndrome. Am J Respir Crit Care Med 2007; 175: 1044 - 1053

17 Zbar B, Alvord WG, Glenn G et al. Risk of renal and colonic neoplasms and spontaneous pneumothorax in the Birt-Hogg-Dubé syndrome. Cancer Epidemiol Biomarkers Prev 2002; 11: 393-400

18 Houweling AC, Gijezen LM, Jonker MA et al. Renal cancer and pneumothorax risk in Birt-Hogg-Dubé syndrome; an analysis of 115 FLCN mutation carriers from 35 BHD families. Br J Cancer 2011; 105: 1912 1919

19 Babaei Jandaghi A, Daliri S, Kikkawa M et al. The discovery of a Persian family with a form of Birt-Hogg-Dubé syndrome lacking the typical cutaneous stigmata of the syndrome. Clin Imaging 2013; 37: 111 - 115 